\title{
Reconstruindo o sistema pessoal Proto-Jê
}

\author{
Ana Suelly Arruda Câmara Cabral ${ }^{1}$ \\ Aryon Dall'Igna Rodrigues ${ }^{2}$ \\ Luciana Gonçalves Dourado ${ }^{3}$ \\ Marcelo Pinho de Valhery Jolkesky ${ }^{4}$ \\ Maxwell Gomes Miranda ${ }^{5}$
}

\section{Resumo}

Fundamenta-se uma hipótese de reconstrução das formas pronominais que teriam constituído o sistema pessoal do Proto-Jê, com base em dados de línguas dos três ramos da família Jê - o setentrional, o central e o meridional. De fundamental importância neste estudo é a identificação de princípios que teriam orientado a distribuição das formas reconstruídas com núcleos sintáticos específicos.

Palavras-chave: Reconstrução morfológica, formas pessoais, funções morfossintáticas, família lingüística Jê, Proto-Jê.

\section{Abstract}

We have developed a reconstructive hypothesis on the pronominal forms which would have constituted the Proto-Jê personal system, based on data from languages belonging to the three branches of the Jê family the northern, the central and the southern. In this study it is of fundamental importance the identification of the principles which would have oriented the distribution of the reconstructed proto-forms with specific syntactic nuclei.

Keywords: Morphological reconstruction, personal forms, morphosyntactic functions, Jê linguistic family, Proto-Jê.

\footnotetext{
${ }^{1}$ Professora do Departamento de Linguística do Instituto de Letras, Vice-Coordenadora do Laboratório de Línguas Indígenas e Coordenadora do Núcleo de Estudos da Amazônia do Centro de Estudos Avançados Multidisciplinares da Universidade de Brasília.

${ }^{2}$ Professor emérito da Universidade de Brasília, pesquisador associado do PPGL e Coordenador do Laboratório de Línguas Indígenas.

${ }^{3}$ Pesquisadora associada ao Laboratório de Línguas Indígenas da Universidade de Brasília.

${ }^{4}$ Doutorando em Linguística no PPGL e pesquisador no Laboratório de Línguas Indígenas da Universidade de Brasília.

${ }_{5}^{5}$ Doutorando em Linguística no PPGL e pesquisador no Laboratório de Línguas Indígenas da Universidade de Brasília.
} 


\section{Introdução}

Neste artigo demonstramos uma hipótese de reconstrução morfológica das marcas de pessoa para o Proto-Jê, fundamentando respostas para as seguintes questões: (a) quais as formas pessoais reconstruíveis para um estágio anterior à separação do ramo meridional em relação aos ramos central e setentrional? (b) quais as relações estabelecidas entre formas pessoais específicas e núcleos sintáticos no Proto-Jê? (c) quais os princípios que teriam norteado a distribuição das formas pessoais na sintaxe dessa protolíngua? (d) quais as principais mudanças ocorridas em línguas particulares com respeito ao sistema de alinhamento original? (e) qual a contribuição que o estudo histórico das mudanças ocorridas nas línguas Jê pode oferecer para o conhecimento das direções e naturezas de mudanças morfossintáticas ocorridas em línguas indígenas brasileiras e que alimentam a discussão de, entre outros, temas como ergatividade, em uma perspectiva histórica? Este artigo é uma versão ampliada do trabalho apresentado durante o IV MacroJê (Recife, 2005) "Reconstrução do sistema de alinhamento Proto-Jê". Os dados $^{6}$ utilizados neste estudo provêm das seguintes línguas e respectivas fontes bibliográficas: Canela-Krahô (Popjes e Popjes 1986); Krahô (Souza 1997; Rodrigues, Cabral e Miranda 2009; Miranda 2010); Parakatejê (Araújo 1989; Ferreira 2001, 2003); Apaniekrá (Alves 2004); Mebengokré (Reis e Silva 2001; Costa 2003; Rodrigues, Cabral e Costa 2004; Cabral e Costa 2004); Apinajé (Ham 1961; Ham et al. 1979; Albuquerque 2004; Oliveira 2005); Suyá (Santos 1997, 2007); Panará (Dourado 1993, 2001, 2002a, 2002b); Xavante (McLeod e Mitchell 2003 [1977]; Santos 2008); Kaingáng (Kindell 1982; Wiesemann 1986; Jolkesky 2010a, b, c); Jaikó (Martius 1867), Xokléng (Urban 1985; Gakran 2005; Jolkesky 2010c).

\section{Hipótese sobre as formas que teriam constituído o sistema pessoal Proto-Jê}

Os dados lingüísticos comparados neste estudo fundamentam consistentemente a reconstrução de duas séries de formas pessoais, uma para

\footnotetext{
$\overline{{ }^{6} \text { Abreviaturas: } \mathrm{ABS}}=$ absolutivo; $\mathrm{ACT}=$ active; $\mathrm{ADV}=$ adverbial; $\mathrm{ALA}=$ alativo; $\mathrm{ASSOC}=$ associativo; $\mathrm{AUX}=$ auxiliar; $\mathrm{BEN}=$ benefactivo; $\mathrm{CAUS}=$ causativo; $\mathrm{COP}=$ cópula; $\mathrm{DEM}=$ demonstrativo; $\mathrm{ENF}=$ enfático; $\mathrm{ERG}=$ ergativo; $\mathrm{EST}$ = estativo; $\mathrm{EVD}$ = evidencial; $\mathrm{FUT}$ = futuro; IMP = imperfective; INES = inessivo; INTERR = interrogativo; IRR = irrealis; MODIF = modificador; N.FUT = não.futuro; NEG = negação; $\mathrm{NOM}=$ nominativo; $\mathrm{NLZ}=$ nominalizador; PAUC = paucal; $\mathrm{PL}=$ plural; $\mathrm{POSP}=$ posposição; $\mathrm{PRF}=$ perfective; $\mathrm{R} 1$ = prefixo relacional de contiguidade; $\mathrm{R} 2=$ prefixo relacional de não contiguidade; $\mathrm{REAL}=$ modo realis; $\mathrm{REFLX}=$ reflexivo; $\mathrm{SBJ}=$ subject; $\mathrm{SG}$ $=$ singular; $\mathrm{SIT}=$ situacional; $\mathrm{ST}=$ stative $; \mathrm{SUBORD}=$ subordinação; $\mathrm{TOP}=$ tópico; $\mathrm{TR}=$ transitivo.
} 
o Proto-Jê, a série B - constituída de formas dependentes -, e outra para o ancestral comum aos ramos setentrional e central, a série A - constituída de formas independentes. A exclusão de línguas meridionais por falta de morfemas cognatos restringe a reconstrução da série A para o ancestral mais imediato das línguas setentrionais e centrais.

\section{Formas pessoais independentes do Proto-Jê setentrional e central}

\begin{tabular}{|l|c|}
\hline \multicolumn{2}{|c|}{ Série A } \\
\hline $1 \pm 3$ & ${ }^{\mathrm{w}} \mathrm{wa} /{ }^{\mathrm{pa}}$ \\
\hline $1+2$ & ${ }^{*} \mathrm{ku}$ \\
\hline $2 \pm 3$ & ${ }^{\mathrm{k} a}$ \\
\hline
\end{tabular}

As formas pronominais das respectivas línguas ${ }^{7}$ que fundamentam a reconstrução dessas proto-formas são dadas a seguir:

Série A

\begin{tabular}{|c|c|c|c|c|c|}
\hline & Ca-Kr & Pk & Su & Apk & Apj \\
\hline $\mathbf{1} \pm \mathbf{2}$ & pa & mpa $(1 \pm 2 \pm 3)$ & & & pa $(1 \pm 2 \pm 3)$ \\
\hline $\mathbf{1} \pm \mathbf{3}$ & wa & wa & wa & wa & \\
\hline $\mathbf{2} \pm \mathbf{3}$ & $\mathbf{k a}$ & $\mathbf{k a}$ & $\mathbf{k a}$ & $\mathbf{k a}$ & $\mathbf{k a}$ \\
\hline $\mathbf{3} \pm \mathbf{3}$ & $\emptyset$ & $\emptyset ; \mathbf{t a}(\mathrm{DEM})$ & $\emptyset ; \mathbf{t a}$ & $\emptyset$ & $\emptyset$ \\
\hline $\mathbf{1}+\mathbf{2}$ & $\mathbf{k u}$ & $\mathbf{k u}$ & $\mathbf{k u}$ & $\mathbf{k u}$ & \\
\hline
\end{tabular}

\begin{tabular}{|c|c|c|c|c|c|c|}
\hline & Me & Pa & Xa & Kg & Xo & P-Jê \\
\hline $\mathbf{1} \pm \mathbf{2}$ & ba $(1 \pm 2)$ & pa $(1+2 \pm 3)$ & & & & $*$ pa \\
\hline $\mathbf{1} \pm \mathbf{3}$ & & & wa $(1 \pm 2 \pm 3)$ & & & ${ }^{*} \mathbf{w a}$ \\
\hline $\mathbf{2} \pm \mathbf{3}$ & ga & ka & $\mathbf{a}(2 \pm 2 \pm 3)$ & & & ${ }^{*} \mathbf{k a}$ \\
\hline $\mathbf{3} \pm \mathbf{3}$ & $\emptyset$ & $\{\mathbf{m a}\} \mathbf{r a}$ & ta & ta & ta & $* \emptyset ;$ *ta \\
\hline $\mathbf{1 + 2}$ & gu $(1+3)$ & & & & & $*$ ku \\
\hline
\end{tabular}

Antes de prosseguirmos, fazemos duas ressalvas importantes, uma sobre as línguas Jê setentrionais e a outra sobre o Xavante, que é Jê central. Em Jê setentrional o sistema pessoal reflete uma dualidade inerente, em que falante e ouvinte alternam-se enquanto elementos focalizados: individualmente, quando um se opõe ao outro, ou dualmente, quando ambos são + focais. Dado

${ }^{7}$ Línguas: $\mathrm{Ca}-\mathrm{Kr}=$ Canela-Krahô; $\mathrm{Pk}=$ Parkatejê; $\mathrm{Su}=$ Suyá; $\mathrm{Apk}=$ Apãniekrá; $\mathrm{Apj}=$ Apinajé; $\mathrm{Me}=$ Mebengokré; $\mathrm{Pa}=$ Panará; $\mathrm{Xa}=$ Xavante; $\mathrm{Kg}=$ Kaingang; $\mathrm{Xo}=$ Xokleng; P-Jê = Proto-Jê. 
o fato de a terceira pessoa ser menos focal, esta pode ser referida quando ego, alter ou ambos estão em foco (Rodrigues e Cabral, ms). Foco é aqui utilizado no sentido empregado por Rodrigues (1990, [1980] 2010) em sua análise das formas pessoais do Tupinambá. Esse é o sentido de que formas pessoais concentram a atenção do ouvinte para distinguir o referente mais importante no evento - dinâmico ou estático - expresso pelo predicado.

Em Xavante, cada forma pessoal pode incluir outro ou muitos e, como são três as formas pessoais, quando combinadas com as partículas quantificadoras somam o total de nove possibilidades. Essa particularidade do Xavante é também observada em Jê Setentrional, o que constitui um traço comum aos sistemas pessoais do Jê setentrional e do Jê central.

\section{Funções argumentais das formas da série $\mathbf{A}$}

Em todas as línguas Jê setentrionais e centrais as formas desta série codificam, em princípio, $A$ e $S$ em orações declarativas independentes.

\section{Xavante}

1) $\begin{array}{ll}\boldsymbol{w a} & \text { mo } \\ 1 & \text { ir }\end{array}$

'Eu vou' (McLeod e Mitchell 1977:18)

2) aro wa tsum ni arroz 1 socar DUAL

'Nós duas estamos socando arroz' (McLeod e Mitchell 1977:38)

3) wa dza ai-pawapto

1 FUT 2-ajudar

'Eu ajudarei você' (McLeod e Mitchell 1977:126)

\section{Suyá}

4) way tute pi

$1 \mathrm{PC}$ arco pegar

'Nós pegamos o arco' (Santos 1997:50)

5) wa ggre

1 dançar

'Eu dancei' (Santos 1997:69)

6) $\quad \begin{array}{lll}p a(n) & w a & t \tilde{\varepsilon} \\ 1 & 1 & \text { ir }\end{array}$

'Eu que fui' (Santos 1997:69) 


\section{Canela-Krahô}

$\begin{array}{llll}\text { 7) wa } & \text { ha } & \text { pixô jũhkà } \\ 1 & \text { FuT } & \text { fruit buy }\end{array}$

'I will buy fruit' (Popjes e Popjes 1986:129)

8) jũ $\mathrm{kam} \quad \mathrm{ka}$ tẽ? pur wỳr

where 22 go? field to

'Where are you going? to the field' (Popjes e Popjes 1986:140)

\section{Xikrín (Mebengokré)}

9) $g a \quad$ ne $\quad g a \quad$ tim

$2 \pm 3 \quad$ PERF $2 \pm 3$ cair

'Vocês caíram' (Costa, notas de campo, 2008)

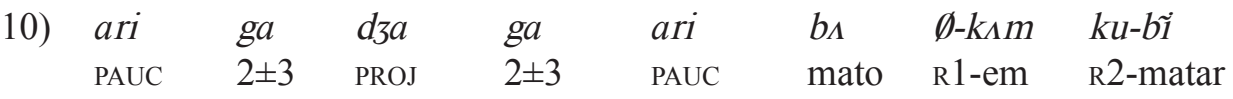
'Vocês o mataram no mato'(Costa, notas de campo, 2008)

11) $\quad b a \quad d z a \quad b a \quad$ тиа

$1 \pm 3 \quad$ PROJ $1 \pm 3$ chorar

'Eu vou chorar' (Costa, notas de campo, 2008)

\section{Panará}

12) $\mathrm{ka}$

$\begin{array}{lllllll}k a & k a & =t i & =r a & =k u i & \tilde{I} k y \tilde{e} & k \hat{o} \\ \text { você.ABS } & \text { IRLS } & =2 \text { SG.NOM } & =1 \text { sg.ABS } & =\mathrm{ir} & \mathrm{eu} & \mathrm{com}\end{array}$

'Você irá comigo' (Dourado 2001:89)

13) $\tilde{i} k y \tilde{e} \quad h \tilde{e} \quad r e \quad \emptyset$-ãpũ $\quad k a$

eu ERG 1sG.ERG REAL.TR 2.ABS-ver você.ABS

'Eu vi você' (Dourado, notas de campo, 1990)

14) $\operatorname{pr} \tilde{e} \quad \emptyset \quad=k a \quad=s-\tilde{a} p \tilde{u} \quad k a \quad h \tilde{e}$

quem.ABS REAL.TR $=2$ SG.ERG $=3$.ABS-ver você ERG

'Quem você viu?' (Dourado 2001:89)

\section{Formas pessoais dependentes do Proto-Jê}

Os dados das línguas comparadas permitem a reconstrução de uma série de formas pessoais para o Proto-Jê, a qual chamamos de série B, mas que, diferentemente da série $\mathrm{A}$, aplica-se à família Jê como um todo e pode ter sido a única série pessoal na proto-língua. A série B é também constituída de três formas, semanticamente correspondentes às da série $\mathrm{A}$ : 


\begin{tabular}{|c|c|}
\hline \multicolumn{2}{|c|}{ Série B } \\
\hline $1 \pm 3$ & $*_{\mathrm{i}}$ \\
\hline $1+2$ & $*(\mathrm{ku}) \mathrm{pa} / \mathrm{wa}$ \\
\hline $2 \pm 3$ & $*_{\mathrm{a}}$ \\
\hline
\end{tabular}

Reflexos dessas formas nas línguas Jê são os seguintes:

Série B

\begin{tabular}{|c|c|c|c|c|c|c|}
\hline & Ca-Kr & Pk & Apk & Apj & Me & Su \\
\hline $\mathbf{1} \pm \mathbf{3}$ & i & i & i & i $\sim$ it $\int$ & i & i \\
\hline $\mathbf{1 + 2}$ & pa & mpa & pa & & guba (1+3) & (k)wa (1pl) \\
\hline $\mathbf{2} \pm \mathbf{3}$ & a & a & a & a & a & a \\
\hline 3 & & $\emptyset$ & & $\emptyset$ & $\emptyset$ & $\emptyset / \mathbf{s}-$ \\
\hline
\end{tabular}

\begin{tabular}{|c|c|c|c|c|c|}
\hline & $\mathbf{P a}$ & $\mathbf{X a}$ & Kg & Xo & P-Jê \\
\hline $1 \pm 3$ & $(\mathbf{i} \sim \mathbf{i}))$ & ii (1) & in (1) & ẽn (1) & $*_{\mathbf{i}}$ \\
\hline $1+2$ & pa (1pl) & wa (1pl) & & & *pa \\
\hline $2 \pm 3$ & a há & $a i \sim a(2 \pm 2)$ & $\tilde{\mathrm{a}}$ & a $(2 \pm 2)$ & $* a$ \\
\hline 3 & $\emptyset$; ti (série A) & $\emptyset ; \mathbf{t i}$ & ti & ti & $* \emptyset ; * t i$ \\
\hline
\end{tabular}

\section{Funções argumentais das formas da série B}

Exemplos contendo reflexos das proto-formas Jê $* i, * p a$ e *a através das línguas, apontam para os seguintes padrões de alinhamento:

\begin{tabular}{|c|c|c|c|}
\hline Alinhamento & \multicolumn{2}{|l|}{ Condição } & Línguas \\
\hline \multirow{2}{*}{$\begin{array}{l}\text { Série } A= \\
\text { Nominativa } \\
(A \text { e } S)\end{array}$} & \multirow{2}{*}{$\begin{array}{l}\text { orações } \\
\text { independentes }\end{array}$} & $\begin{array}{l}\text { não modificadas por } \\
\text { expressão adverbial }\end{array}$ & $\mathrm{Me}, \mathrm{Su}, \mathrm{Xa}$ \\
\hline & & tempo futuro & $\mathrm{Ca}-\mathrm{Kr}$ \\
\hline \multirow{3}{*}{$\begin{array}{l}\text { Série B = } \\
\text { Absolutiva } \\
(\mathrm{S}, \mathrm{O}, \mathrm{OI}, \text { Poss) }\end{array}$} & \multicolumn{2}{|c|}{$\begin{array}{l}\text { predicados negados e } \\
\text { em orações dependentes }\end{array}$} & $\begin{array}{l}\mathrm{Me}, \mathrm{Su}, \mathrm{Xa}, \mathrm{Apj}, \\
\mathrm{Apk}, \mathrm{Ca}-\mathrm{Kr}\end{array}$ \\
\hline & \multicolumn{2}{|c|}{$\begin{array}{l}\text { predicados modificados por } \\
\text { expressão adverbial }\end{array}$} & $\begin{array}{l}\mathrm{Me}, \mathrm{Su}, \mathrm{Xa}, \mathrm{Apj} \\
\text { Apk, Ca-Kr }\end{array}$ \\
\hline & \multicolumn{2}{|l|}{ tempo passado } & $\mathrm{Ca}-\mathrm{Kr}$ \\
\hline \multirow{2}{*}{$\begin{array}{l}\text { Série } \mathrm{B}+\text { te/je/re } \\
=\text { Ergativa }(\mathrm{A})\end{array}$} & \multicolumn{2}{|c|}{$\begin{array}{l}\text { predicados negados, predicados } \\
\text { modificados por expressão adverbial }\end{array}$} & $\begin{array}{l}\mathrm{Me}, \mathrm{Su}, \mathrm{Ca}-\mathrm{Kr} \\
\mathrm{Apk}\end{array}$ \\
\hline & \multicolumn{2}{|l|}{ tempo passado } & $\mathrm{Ca}-\mathrm{Kr}$ \\
\hline
\end{tabular}




\section{Alinhamento das formas das séries A e B}

Exemplos que mostram a distribuição dos reflexos da série $\mathrm{B}$ reconstruída para o Proto-Jê, nas línguas modernas da família Jê, são apresentados em seguida:

\section{Suyá}

\section{Predicados negados}

15) i-ngrere kere

1PS-dançar NEG

'Eu não danço' (Santos 1997:66)

16) hwararo i-ngrere kere

ontem 1PS-dançar NEG

'Ontem eu não dancei' (Santos 1997:52)

17) i-re hwïgro y-antoro kere

1s-ERG lenha REL-pendurar NEG

'Eu não pendurei a lenha' (Santos 1997:56)

\section{Predicados modificados por outras expressões adverbiais}

18) hẽn kwa ngrere ro ta ASP $1+2$ dançar POSP verbo.posicional

'Nós estamos dançando' (Santos 1997:52)

19) i-ngrere mã

1PS-dançar POSP

'Eu dançarei' (Santos 1997:52)

20) i-re hwĩsi ren mã

1PS-MS fruta colher POSP

'Eu vou colher a fruta' (Santos 1997:52)

\section{Xavante}

\section{Predicados negados}

21) $\pi$ mori $\tilde{o} d i$

1 ir NEG

'Não vou' (McLeod e Mitchell 1977:81) 


\section{Predicados modificados por outras expressões adverbiais}

22) wa dza morĩ tsu

1 PROJ ir depressa

'Irei depressa' (McLeod e Mitchell 1977:133)

23) te dza $\dddot{1}$ mori $t s u$

1 PROJ 1 ir depressa

'Irei depressa' (McLeod e Mitchell 1977:133)

\section{Orações dependentes}

24) $\dddot{1}$ mori waptsi wa dza romhu

1 ir quando 1 PROJ trabalhar

'Quando eu for, vou trabalhar' (McLeod e Mitchell 1977:49)

25) buru $u$ ĩi morĩ wamhã wa dza wadzo roça para 1 ir se 1 PROJ capinar

'Se eu for à roça, vou capinar' (McLeod e Mitchell 1977:53)

26) İ-sisanawã ma tewe ãwisi uniãa a-simaĩwe

1-irmão de.longe trouxe arco 2-quer

'Meu irmão trouxe o arco que você quer' (Rafael Wederó'ó'wa We'réé)

\section{Canela/Krahô}

\section{Predicados negados ${ }^{8}$}

27) a-mõr nare

2-go NEG

'You did not go' (Popjes e Popjes 1986:132)

28) $\mathrm{ka}$ ha ajkahu kakrô $\mathrm{ka}$ ha a-kator nare

2 FUT run but 2 FUT 2-arrive NEG

'You will run but you will not arrive' (Popjes e Popjes 1986:149)

\section{Predicados no passado}

29) a-te po kuran

2-PAST deer kill

'You killed a deer' (Popjes e Popjes 1986:153)

\footnotetext{
${ }^{8}$ Há, no entanto, alguns contextos sintáticos, como no caso em que o foco da negação é o sintagma instrumental e não o predicado (Popjes e Popjes 1986:161), em que o padrão de alinhamento é nominativo:

$\begin{array}{lllllll}\text { katõk } & \text { to } & \text { Wa } & \text { ha } & \text { po } & \text { kuran } & \text { nare } \\ \text { gun } & \text { INSTR } & 1 & \text { FUT } & \text { deer } & \text { kill } & \text { NEG } \\ \text { 'I won't kill the deer with a gun' (Popjes e Popjes } & 1986: 161) & \end{array}$
}


30) $a-j \tilde{o} t$

2-sleep

'You slept' (Popjes e Popjes 1986:132)

\section{Predicados modificados por outras expressões adverbiais}

31) i-te i-jõ krẽr partu

1-PAST 1-food eat completely

'I ate all my food completely' (Popjes e Popjes 1986:182)

32) mẽ par-te põhi $\emptyset-k e \tilde{e} k^{h} \tilde{e}-n \quad k r i n a r \varepsilon$

PL $1 \pm 3$-POSP milho $\mathrm{R}^{1}$-quebar-NLZ muito

'Nós colhemos (quebramos) muito milho' (Miranda 2010:51)

\section{Orações dependentes}

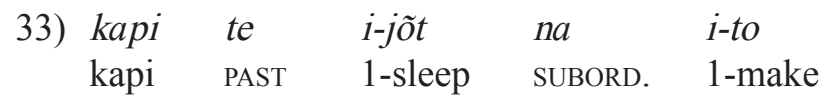

'Kapi made me sleep' (Popjes e Popjes 1986:143)

34) i-te i-prõ jàpên na ton

1-PAST 1-wife work SUBORD. 3+make

'I made my wife work' (Popjes e Popjes 1986:143)

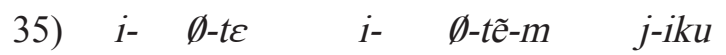

1- R ${ }^{1}$-POSP $\quad 1-\quad \mathrm{R}^{1}$-ir-NLZ $\mathrm{R}^{1}$-parar

'Eu parei de ir (andar)' (Miranda 2010:69)

36) i-te hũmre te rop kuran ita pupun

1-PAST man PAST dog kill DEM see

'I saw the man who killed the dog' (Popjes e Popjes 1986:171)

\section{Xikrín (Mebengokré)}

\section{Predicados negados}

37) $i \quad \emptyset$-tẽm ket nũ

$2 \pm 3 \quad R^{1}$-ir NEG ainda

'Eu ainda não fui' (Costa, notas de campo, 2009)

38) $g a \quad$ a $\quad \emptyset$-tor $\quad$ ket

$2 \pm 3 \quad 2 \pm 3 \quad$ R1- dançar NEG

'Você não dançou' (Costa, notas de campo, 2009) 
39) $\begin{array}{lllll}a & \emptyset \text {-je } & i & \emptyset \text {-kakje-re } & \text { ket } \\ 2 \pm 3 & \mathrm{R}^{1} \text {-por } & 1 \pm 3 & \mathrm{R}^{1} \text {-beliscar-NLZ } & \text { NEG }\end{array}$

'Você não me beliscou' (Costa, notas de campo, 2009)

\section{Predicados modificados por outras expressões adverbiais}

40) $\quad b a \quad i \quad$ Ø-prere $\quad$ kumej

$1 \pm 3 \mathrm{ENF} \quad 1 \pm 3 \quad \mathrm{R}^{1}$-cantar muito

'Eu canto muito' (Costa, notas de campo, 2009)

41) $\quad$ i $\quad$-je $\quad$ mebẽgokre $\emptyset$-kabẽn mari prire

$1 \mathrm{SG} \mathrm{R}^{2}$-ERG xikrín $\mathrm{R}^{2}$-falar saber pouco

'Eu sei falar um pouquinho de Xikrín' (Costa, notas de campo, 2009)

\section{Orações dependentes}

42) $\quad b a \quad$ ne $\quad b a \quad i \quad \emptyset_{-1} \tilde{\Lambda} \quad\left[\begin{array}{lll}a & -\tilde{o}-t] & \text { pr } \Lambda m\end{array}\right.$ $1 \pm 3$ ENF N.FUT $1 \pm 3 \quad 1 \pm 3 \quad \mathrm{R}^{1}$-para $\quad\left[2 \pm 3 \quad \mathrm{R}^{1}\right.$-dormir-NLZ $]$ querer 'Eu quero que você durma' (Costa, notas de campo, 2009)

43) $\quad$ i $\quad \emptyset$-bım nẽ $\quad k u-t \varepsilon \quad\left[i \quad \emptyset_{-j \varepsilon} \quad\right.$ tep $\left.\quad \emptyset-k r \tilde{e}-n\right] \quad$ pr $]$ $1 \pm 3 \quad \mathrm{R} 1$-pai N.FUT $\mathrm{R}^{2}$-por $\left[1 \pm 3 \mathrm{R}^{1}\right.$-por peixe $\mathrm{R}^{1}$-comer-NLZ $]$ querer 'Meu pai quer que eu coma peixe' (Costa, notas de campo)

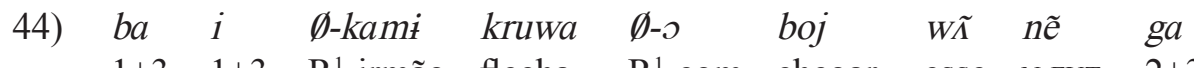
$1 \pm 3 \quad 1 \pm 3 \quad \mathrm{R}^{1}$-irmão flecha $\mathrm{R}^{1}$-com chegar esse N.FUT $2 \pm 3$

$\left[\begin{array}{llll}a & \emptyset \text {-je } & \emptyset \text {-biri- } \emptyset & m \tilde{\Lambda}\end{array}\right]$

$\left[2 \pm 3 \quad \mathrm{R}^{1}\right.$-por $\mathrm{R}^{2}$-comprar-NLZ MODIF

'Meu irmão trouxe a flecha que você quer comprar' (Costa, notas de campo)

\section{Línguas divergentes}

\section{- Panará, Kaingáng e Xokléng}

O Panará, de acordo com Dourado (2001), possui três séries de clíticos pronominais, uma nominativa, uma ergativa e uma absolutiva, assim distribuídas:

\begin{tabular}{|l|l|l|}
\hline NOMINATIVA & $S$ e $A$ & Modo irrealis \\
\hline ERGATIVA & $A$ & Modo realis \\
\hline ABSOLUTIVA & $S$ e $O$ & Modo realis \\
\hline
\end{tabular}




\section{A, modo realis}

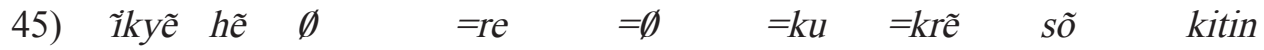
1SG ERG REAL.TR 1SG.ERG 3SG.ABS =AUX =comer comida pouca 'Eu comi pouca comida' (Dourado 2001:52)

46) $\operatorname{pr} \tilde{\mathrm{e}} \quad \emptyset \quad=k a \quad=S \quad-\tilde{p} p \tilde{u} \quad k a \quad h \tilde{e}$ quem.ABS REAL.TR $=2$ SG.ERG $=3 . \mathrm{ABS}$-ver você ERG

'Quem você viu?' (Dourado 2001:89)

\section{$\mathrm{S}$, modo realis}

47) $\begin{array}{lll}\text { yi } & =r a \quad=t i \quad a k u a\end{array}$

REAL.INTR $=1$ SG.ABS $=$ morrer EVD

'Eu quase morri/eu desmaiei' (Dourado 2001:63)

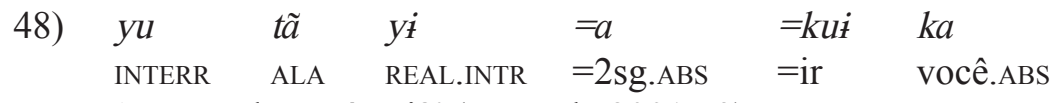
'Para onde você vai?' (Dourado 2001:59)

\section{S, modo irrealis}

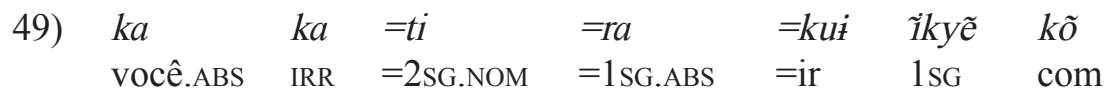
'Você irá comigo' (Dourado 2001:89)

\section{S e O, independentemente de modo}

50) $\quad$ yi $\quad p a \quad p o$ REAL.INTR 1PL.ABS chegar

'Nós chegamos' (Dourado, notas de campo, 1988)

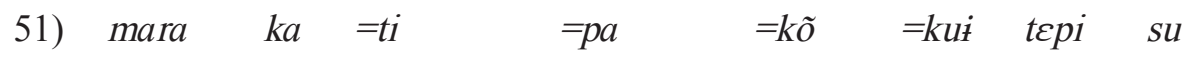
3SG.ABS IRR $=3 \mathrm{sG} . \mathrm{NOM}=1 \mathrm{PL} . \mathrm{ABS}=\mathrm{com}=\mathrm{ir}$ peixe alvo 'Ele vai pescar conosco' (Dourado, notas de campo, 1988)

52) $\quad p a \quad=j o ̃ t i \quad$ îkieti $k a \quad=p a \quad$ tẽ 1pl.ABS =dormir muito IRR $=1 \mathrm{pl} . \mathrm{ABS}$ ir 'Nós ficamos aqui uns dias e depois vamos viajar' (Dourado, notas de campo, 1988)

\section{Reflexos do P-Jê *i}

53) $\tilde{i} k y \tilde{e} \quad h \tilde{e} \quad \emptyset \quad$ re $\quad \emptyset \quad$ pi-ri $\quad$ atõsi eu ERG REAL.TR 1ERG 3SG.ABS comprar-PERF munição 'Eu comprei munição' (Dourado, notas de campo, 1988) 
54) $\tilde{i}$ kow tã

1 rio ALA

'Eu (vou) para o rio' (Dourado 2001:45)

55) $\tilde{a}$ kow tã

2 rio ALA

'Você (vai) para o rio' (Dourado 2001:45)

O Kaingáng aparentemente é a única língua Jê que possui uma só série pessoal:

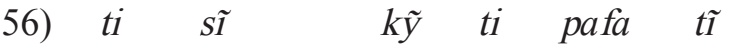

he be.small SEG he nurse IMP

'When he is small he nurses' (Kindell 1982:72)

57) hã ra ẽg cavalo ko vã nỹgtĩ thus in.spite.of we horse eat NEG ST 'However, we do not eat horses' (Kindell 1982:72)

58) ẽg tĩgja kar mĩ ẽg tóg our going all around we SBJ ẽg pẽn ki rĩrtĩ $k e$ tũ nĩ mũ

our foot in care IMP do NEG ST PRF ra ẽg tóg pó tỹ krĩm kenh mũ in.spite.of we SBJ rock by hurt FUT PRF

'Whenever we go we watch our feet; if we don't we will hurt them on the rocks' (Kindell 1982:219)

59) vãs recently FUNAI ACT me summon PRF i mréke ũ mré my colleague other with

'Recently FUNAI summoned me, along with one of my colleagues' (Kindell 1982:235)

\section{Marcas discursivas}

60) $i \quad \tilde{i} n \quad v \tilde{y}$ pũr mỹr

1 house TOP burn verily

'My house burned down really' (Kindell 1982:69)

61) vẽ kuprig tóg nỹgtĩ gé sir REFLX spirit SBJ be.ST also then

'There are spirits also' (Kindell 1982:69) 


\section{Jeikó (Único representante do subconjuto Nordeste)}

\section{Verbos}
62a) ti loschung
3o assar
62b) ti kua
'assá-lo'
3o comer
62c) ti uing
30 matar
'comê-lo'
'matá-lo' (Martius 1867:143)

\section{Nomes}

\begin{tabular}{|c|c|c|c|c|c|}
\hline 63a) & $\begin{array}{l}\text { ae pang } \\
2 \text { braço } \\
\text { 'braço' }\end{array}$ & 63b) & $\begin{array}{l}\text { ay anté } \\
2 \text { dente } \\
\text { 'dente' }\end{array}$ & 63c) & $\begin{array}{l}\text { ae croh } \\
2 \text { coxa } \\
\text { 'coxa' }\end{array}$ \\
\hline 63d) & $\begin{array}{l}\text { ae netta } \\
2 \text { língua } \\
\text { 'língua' }\end{array}$ & 63e) & $\begin{array}{l}\text { ae jusi } \\
2 \text { seio } \\
\text { 'seio' }\end{array}$ & $63 \mathrm{f})$ & $\begin{array}{l}\text { ae na enong } \\
2 \text { mão } \\
\text { 'mão' }\end{array}$ \\
\hline $63 \mathrm{~g})$ & $\begin{array}{l}\text { ae reng } \\
2 \text { pênis } \\
\text { 'pênis' }\end{array}$ & 63h) & $\begin{array}{l}\text { ae oaénũ } \\
2 \text { vagina } \\
\text { 'vagina' }\end{array}$ & 63i) & $\begin{array}{l}\text { ae ingko } \\
2 \text { boca } \\
\text { 'boca' }\end{array}$ \\
\hline
\end{tabular}

63j) ae pähno

2 pé

'pé' (Martius 1867:143)

\section{As subcategorias de número}

As línguas da família Jê manifestam uma distinção entre singular/dual, paucal e plural (Mebengokré, Apinajé), singular, paucal e plural (Suyá), singular, dual, paucal e plural (Panará), singular, dual e plural (Xavante) ou singular e plural (Xokleng). As marcas de paucal e de dual das línguas Jê são reflexos do morfema *ari $\sim *^{*} a j$ reconstruível para o Proto-Jê, e na maioria das línguas da família, a marca de plural/associativo são reflexos do morfema *mẽe, também reconstruível para o Proto-Jê.

\begin{tabular}{|c|c|c|c|c|c|c|c|c|c|c|c|c|c|}
\hline & & Me & Apk & Pk & Kr & Apj & \multicolumn{2}{|c|}{ Su } & \multicolumn{2}{|c|}{ Xa } & Kg & Xo & Pa \\
\hline & & & & & & Série A & Série B & Série A & Série B & & & \\
\hline DUAL & $1 \mathrm{a}$ & & & & & & & $\ldots$..ni & $\ldots$ (wa)'wa & & mẽ \\
\hline & $2 \mathrm{a}$ & & & & & & & $\ldots$ wa'wa & & & & mẽ \\
\hline & $3 \mathrm{a}$ & & & & & & & $\begin{array}{l}\text {...dza'ra/ } \\
\text {...dzahuré }\end{array}$ & ...dzahuré & & mẽ \\
\hline
\end{tabular}




\begin{tabular}{|l|c|c|c|c|c|c|c|c|c|c|c|c|c|}
\hline PAUCAL & $1 \mathrm{a}$ & ari & & & & $\ldots$..ai & & & & & & \\
\hline & $2 \mathrm{a}$ & aj & & & & $\ldots$ ai & & & & & \\
\hline & $3 \mathrm{a}$ & ari & & & $\ldots$..je & & & & & -pyira \\
\hline
\end{tabular}

O Xavante apresenta uma concordância de padrão absolutivo na marcação de número, segundo o argumento interno de verbos transitivos e intransitivos. McLeod e Mitchell (1977:213-214) descrevem para o Xavante temas de verbos transitivos e intransitivos com formas supletivas, cujas formas singulares, duais e plurais se combinam com $\mathrm{S}$ e $\mathrm{O}$, com referentes que são respectivamente singular, paucal e plural:

$\begin{array}{lllll}\text { 6ede } & ? w a & \emptyset & d z a & \text { ti-Po } \\ \text { pau }_{\mathrm{i}} & 1 & \text { POT } & \text { PROJ } & 3_{\mathrm{i}} \text {-levar }\end{array}$

'Eu vou levar o pau'

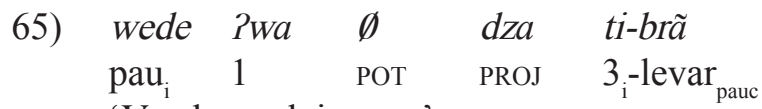

'Vou levar dois paus'

66) wede Pwa $\emptyset \quad d z a \quad$ Pwa-tsiwi $\emptyset$-Pəri di

$\begin{array}{llllll}\mathrm{pau}_{\mathrm{i}} & 1_{\mathrm{j}} \quad \text { POT PROJ } & 1_{\mathrm{j}} \text {-muitos } & 3_{\mathrm{i}} \text {-levar } & \text { NLZ }\end{array}$

'Nós (plural) vamos levar pau'

67) wede Pwa $\emptyset \quad d z a \quad \emptyset$-brã bĩ $\quad d i$

$\begin{array}{llllll}\mathrm{pau}_{\mathrm{i}} & 1 & \text { POT } & \text { PROJ } & 3_{\mathrm{i}} \text {-levar } & \\ \text { pauc } & \text { NLZ }\end{array}$

'Nós dois vamos levar dois paus'

68) wede $P w a \quad \emptyset \quad d z a \quad \emptyset$-wajbu

$\begin{array}{lllll}\mathrm{pau}_{\mathrm{i}} & 1 & \text { POT } & \text { PROJ } & 3_{\mathrm{i}} \text {-levar }_{\mathrm{pl}}\end{array}$

'Vou levar os paus (plural)'

69) wede $2 w a \quad \emptyset \quad d z a \quad \emptyset$-wajbu dï

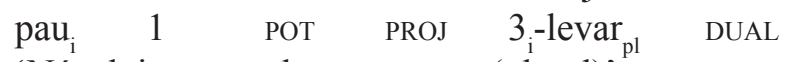

'Nós dois vamos levar os paus (plural)' 


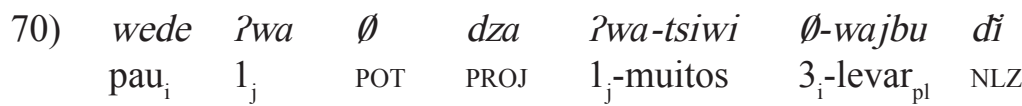
'Nós (plural) vamos levar os paus (plural)'

O Kaingáng e o Xokléng apresentam concordância de padrão absolutivo encontrada nos verbos que distinguem temas singulares e plurais. Urban (1985) descreve o seguinte padrão absolutivo no Xokléng, por meio dos seguintes exemplos:

Concordância com S:

71) ta wũ tẽ mũ

he 3-NOM go.SG ACT

'He went' (Urban 1985:166)

72) วฤ $\quad$ ũ mũ mũ

they 3-NOM go.PL ACT

'They went' (Urban 1985:171)

Concordância com O:

73) ta wũ ti penũ mũ

he 3-NOM he shoot.SG ACT

'He shot him' (Urban 1985:166)

74) ta wũ mẽ on pin mũ

he 3-NOM distributive they shoot.PL ACT

'He shot them' (Urban 1985:176)

\section{As marcas ergativas}

Na maioria das línguas Jê os predicados que têm por núcleo a forma nominal de um verbo transitivo (forma longa), têm o seu agente codificado pela série B combinada com marcas que têm sido descritas como temporais no complexo Timbira, como marca ergativa no Mebengokré e como marca de sujeito de verbo transitivo no Suyá (Santos 1997:56). No Panará, um morfema re é encontrado como marca de primeira pessoa ergativa e um morfema ne alternando com re marca a primeira e terceira pessoa do plural ergativa. É provável que todas essas marcas sejam reflexos da combinação de três antigos morfemas ${ }^{*} j$ - 'relacional de contiguidade', ${ }^{*} t$ - 'relacional de não contiguidade' e a 'posposição' te do Proto-Jê.

\begin{tabular}{|c|c|c|c|c|c|c|c|}
\hline$M e$ & $A p k$ & $P k$ & $K r$ & $A p j$ & $S u$ & $X a$ & $P a$ \\
\hline -je/-te & te & te & te & te & re/te & $($ te $)$ & $($ re/ne) $/$ hẽ \\
\hline
\end{tabular}


Apesar da aparente ocorrência dessas marcas em construções transitivas, fato que tem levado vários lingüistas à interpretação de que são expressões de um caso ergativo na família Jê, há contra-evidências em línguas como o Krahô e o Xavante, nas quais os respectivos morfemas cognatos podem combinar-se com as mesmas marcas pessoais que codificam tanto o sujeito de verbos transitivos, quanto o de verbos intransitivos.

Urban (1975:172) mostra que em Xokléng o morfema tr̃, que marca agentes de verbos transitivos, pode também ocorrer quando o predicado é intransitivo, mas imediatamente seguido por expressão adverbial:

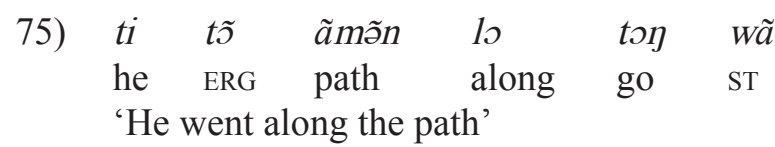

Igualmente, Jolkesky (2010a, b) observa que em Kaingáng o morfema tõ marca não somente agentes de verbos transitivos nas orações de ordem rígida, do tipo SXOV, como as subordinadas e declarativas, mas também sujeitos intransitivos - sejam eles agentes ou pacientes - sempre que houver um argumento indireto (X), por exemplo, um argumento locativo ou adverbial. Em outras palavras, a ocorrência de dois argumentos nestes tipos de oração já implica na obrigatoriedade do marcador, independentemente de relações gramaticais ou transitividade.

$\begin{array}{llll}\text { 76a) } t i \quad \text { há } & \text { ninn } & k \tilde{y} \\ \text { 3.SG bom } & \text { EST } & \text { SIT } \\ \text { 'Ele sendo bom, } \ldots & \end{array}$

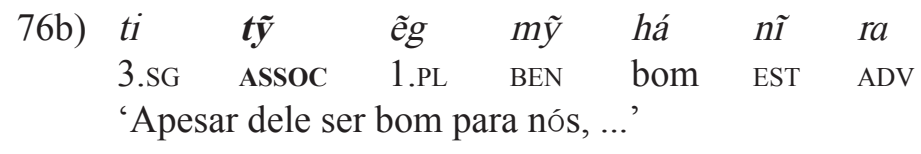

$\begin{array}{lll}\text { 77a) } & t i \quad \text { ter } & k \tilde{y} \\ \text { 3.SG } \quad \text { morrer.SG } & \text { SIT } \\ \text { 'Ele morrendo, ...' } & \end{array}$

77b) is $\quad a$ tugnĩn ter $\quad$ ky

1.SG.ASSOC 2.s CAUS morrer.SG SIT

'Eu morrendo por tua causa, ...'

$\begin{array}{llllll}\text { 77c) } & t i \quad t \tilde{y} & f i & \text { jagfy } & \text { ter } & k \tilde{y} \\ \text { 3.SG } & \text { ASSOC } & \text { 3.F.S } & \text { CAUS } & \text { morrer.SG } & \text { SIT } \\ \text { 'Ele morrendo por ela (no lugar dela), ...' } & \end{array}$


78a) ajjag rĩnrĩr $\quad$ ky

2.PL viver.PL SIT

'Vocês vivendo, ...'

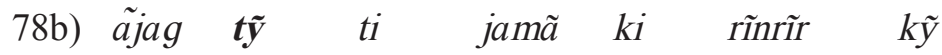

2.PL ASSOC 3.SG local INES viver.PL SIT

'Vocês vivendo na aldeia dele,...'

Jolkesky (2010c) retoma a questão e demonstra que tỹ em Kaingáng e tõ em Xokleng devem ser vistos como posposições prototipicamente associativas que, por desenvolvimento histórico, tiveram seu uso estendido para especificadores: (i) de sintagmas nominativos rebaixados para posição oblíqua e (ii) de sintagmas nominais nas funções adjuntiva, apositiva ou predicativa. Os dados abaixo são do Xokléng.

79a) óg mũ kũ

3.PL ir.PL SIT

'Eles indo...'

79b) óg tõ ẽnh jamã ló mũ kũ

3.PL ASSOC 1.SG local DIR ir.PL SIT

'Eles indo para a minha aldeia...'

80a) katxol vã

cachorro COP

'É o cachorro.'

80b) ẽnh mãg to $\tilde{\boldsymbol{o}}$ katxol vã

1.SG criação ASSOC cachorro COP

'É meu cachorro de estimação.'

81a) ẽnh ji vã

1.SG filho COP

'É meu filho.'

81b) a tõ ẽnh ji vã

2.SG ASSOC 1.SG filho COP

'Você é meu filho.'

Miranda (2010) mostra que em Krahô, o morfema $t \varepsilon$ pode marcar o sujeito de verbos intransitivos em algumas situações nas quais: (i) o sujeito intransitivo é foco/tópico do predicado, sendo expresso 
simultaneamente pela série A e pela série B, sendo esta a que se combina com o morfema $t \varepsilon$, e (ii) predicados verbais intransitivos com um argumento oblíquo que Popjes e Popjes (1986) descreveram como pseudo-transitivos.

\section{Sujeito focalizado/topicalizado}

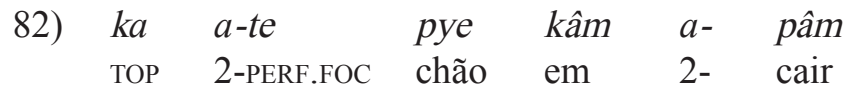

'Você, foi você que caiu no chão' (Souza 1997:33)

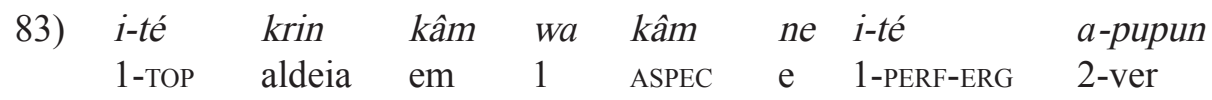

'Eu vim para a aldeia e eu vi você' (Souza 1997:68)

\section{Predicados verbais intransitivos com argumento oblíquo}

$\begin{array}{llllll}\text { 84) mé } & \text { ku-té } & \text { pye } & \text { kâm } & \text { mé } & \emptyset \text {-pâm } \\ \text { PL } & \text { 3-PERF-FOC } & \text { chão } & \text { em } & \text { PL } & \text { S-cair }\end{array}$

‘Eles caíram no chão' (Souza 1997:73)

85) i-te a-mã i- cator

1-PAST 2-to 1- arrive

'I found you (arrived to you)' (Popjes e Popjes 1986:131)

Estudos mais aprofundados sobre o uso destes morfemas poderão revelar um quadro mais exato do real status da ergatividade nas línguas Jê e consequentemente das funções das suas classes pronominais.

\section{Considerações finais}

Neste ensaio apresentamos uma primeira abordagem comparativa da sintaxe das línguas da família Jê, com o intuito de depreender o que há de comum entre elas, como provável reflexo da organização gramatical que estaria presente na respectiva proto-língua. Embora ainda hoje haja muito trabalho comparativo a ser feito com respeito ao léxico e à fonologia, a primeira proposta de reconstrução fonológica publicada por Davis, em 1966, já permitiu assegurar a consistência da família Jê como grupo genético bem definido. As correspondências morfossintáticas mostradas aqui contribuem certamente para a consolidação da noção de família Jê e para um primeiro acesso à gramática de seu ancestral pré-histórico, o Proto-Jê. A divergência maior mais observada no Kaingáng e no Xokléng possivelmente tem a ver com as vicissitudes que o ramo meridional da família deve ter experimentado no decorrer de uma 
longa migração pré-histórica para os campos do sul. A diferenciação também notável do Panará provavelmente decorre de contatos com outros povos numa migração bem mais recente, a que se viram forçados seus falantes a partir do século XVIII e que os levou a deslocar-se da bacia do Tocantins-Araguaia para a do Tapajós a fim de livrarem-se da guerra que lhes moveu a Coroa Portuguesa.

\section{Referências}

Albuquerque, Francisco Edviges. 2004. A estrutura do verbo em Apinayé. Liames 4:51-57. Campinas.

Alves, Flávia de Castro. 2004. O Timbira falado pelos Canela Apaniekrá: uma contribuição aos estudo da morfossintaxe de uma língua Jê. Tese de doutorado, Universidade Estadual de Campinas.

Cabral, Ana Suelly A. Câmara, Lucivaldo Silva da Costa. 2004. Xikrín e línguas Tupí-Guaraní: marcas relacionais. Liames 4:7-19. Campinas.

Cabral, Ana Suelly A. Câmara, Aryon D. Rodrigues, Lucivaldo S. da Costa. 2004. Notas sobre ergatividade em Xikrín. Liames 4:21-28. Campinas.

Cavalcante, Marita Pôrto. 1987. Fonologia e morfologia da língua Kaingáng: o dialeto de São Paulo comparado com o do Paraná. Tese de doutorado, Universidade Estadual de Campinas.

Costa, Lucivaldo Silva da. 2003. Flexão relacional, marcas pessoais e tipos de predicados em Xikrín: contribuição para os estudos sobre ergatividade em línguas Jê. Dissertação de mestrado, Universidade Federal do Pará.

Dourado, Luciana. G. 1992. Concordância em Panará. Anais da 44a Reunião Anual da SBPC, São Paulo, p. 390.

Dourado, Luciana. 2001. Aspectos morfossintáticos da língua Panará (Jê). Tese de doutorado, Universidade Estadual de Campinas.

Dourado, Luciana. 2002a. Ergatividade em Panará. In: Ergatividade na Amazônia I. Brasília: Centre d'études des langues indigènes d'Amérique (CNRS-IRD) e Laboratório de Línguas Indígenas (UnB), pp. 103-108.

Dourado, Luciana. 2002b. A expressão de posse em Panará. In: A. S. A. C. Cabral, A. D. Rodrigues (orgs.), Linguas indígenas brasileiras: fonologia, gramática e história: Atas do I Encontro Internacional do Grupo de Trabalho sobre Linguas Indigenas da ANPOLL, t. I, pp. 98-103. Belém: EDUFPA.

Dourado, Luciana. 2003. Ergatividade e transitividade em Panará. In: Ergatividade na Amazônia II. Brasília: Centre d'études des langues indigènes d'Amérique (CNRS-IRD) e Laboratório de Línguas Indígenas (UnB), pp. 165-172. 
Ferreira, Marília. 2001. Aspectos das classes de palavras em Parkatêjê: uma abordagem tipológico-funcional. In: A. S. A. C. Cabral, Aryon D. Rodrigues (orgs.), Estudos sobre línguas indígenas I. Belém: UFPA, pp 147-166.

Ferreira, Marília. 2003. Estudo morfossintático da língua Parkatêjê. Tese de doutorado, Universidade Estadual de Campinas.

Gakran, Nanblá. 2005. Aspectos morfossintáticos da língua Laklãnõ (Xokleng). Dissertação de mestrado, Universidade Estadual de Campinas.

Ham, Patricia. 1961. Apinayé grammar. Anápolis, GO: Summer Institute of Linguistics.

Ham, Patricia, Helen Waller, Linda Koopmsn. 1979. Aspectos da língua Apinayé. Cuiabá: Sociedade Internacional de Linguística.

Jolkesky, Marcelo Pinho de Valhery. 2010a. Re: ergatividade em línguas Jê setentrionais. Disponível em: $<$ http://br.groups.yahoo.com/group/ etnolinguistica/message/2422>

Jolkesky, Marcelo Pinho de Valhery. 2010b. Re: ergatividade em línguas Jê setentrionais. Disponível em: <http://br.groups.yahoo.com/group/ etnolinguistica/message/2429>

Jolkesky, Marcelo Pinho de Valhery. 2010c. As funções do associativo *tã em Proto-Jê Meridional. Comunicação apresentada no VII Macro-Jê. Laboratório de Línguas Indígenas, Universidade de Brasília.

Jolkesky, Marcelo Pinho de Valhery. 2010d. Reconstrução fonológica e lexical do Proto-Jê Meridional. Dissertação de mestrado, Universidade Estadual de Campinas.

Kindell, Gloria E. 1982. Discourse strategies in Kaingáng literacy materials. $\mathrm{Ph}$. D. dissertation. Georgetown University.

Martius, Karl Friedrich Phillipp von. 1867. Beiträge zur Ethnographie und Sprachenkunde Amerika's zumal Brasiliens. I. Zur Ethnographie, II. Zur Sprachenkunde. Leipzig: Friedrich Fleischer.

McLeod, Ruth, Valerie Mitchell. 1977 Aspectos da lingua Xavante. Cuiabá: Summer Institute of Linguistics.

Miranda, Maxwell Gomes. 2010. As nominalizações na sintaxe da língua Krahô (Jê). Dissertação de mestrado, Universidade de Brasília.

Nimuendajú, Curt. 1932. Die Kayapó des mittleren Xingú. In: mesmo autor, Idiomas indígenas del Brasil. Revista del Instituto de Etnología, tomo II, pp. 552-567. Tucumán: Universidad Nacional de Tucumán.

Oliveira, Christiane Cunha de. 2005. The language of the Apinajé people of Central Brazil. Ph. D. dissertation, University of Oregon. 
Popjes, Jack, Jo Popjes. 1986. Canela-Krahô. In: D. Derbyshire, G. Pullum (orgs.). Handbook of Amazonian languages 1:129-199. Berlim: Mouton de Gruyter.

Reis Silva, Maria Amélia. 2001. Pronomes, ordem e ergatividade em Mebengokré (Kayapó). Dissertação de mestrado, Universidade Estadual de Campinas.

Rodrigues, Aryon D. 1985. Evidence for Tupí-Karíb relationships. In: E. M. Klein, L. R. Stark (orgs.), South American indian languages: retrospect and prospect. Austin: University of Texas Press, pp. 371-404.

Rodrigues, Aryon D. 1986. Linguas brasileiras: para o conhecimento das línguas indigenas. São Paulo: Edições Loyola.

Rodrigues, Aryon D. 1992. Grammatical affinities among Tupí, Karíb, and Macro-Jê, ms.

Rodrigues, Aryon D. 1999. Macro-Jê. In: R. M. W. Dixon, A. Y. Aikhenvald (orgs.), The Amazonian languages, p. 162-206. Cambridge: Cambridge University Press.

Rodrigues, Aryon D. 2001. Flexão relacional no tronco lingüístico Macro-Jê. Boletim da ABRALIN 25:219-231, Fortaleza: Imprensa Universitária.

Santos, Juliana Pereira dos. 2008. Marcas pessoais, concordância de número e alinhamento em Xavante. Dissertação de mestrado, Universidade de Brasília.

Santos, Ludoviko C. dos. 1997. Descrição de aspectos morfossintáticos da língua Suyá/Kisêdjê (Jê). Tese de doutorado, Universidade Federal de Santa Catarina.

Santos, Ludoviko C. dos. 2007. Concordância de número em Kaingáng: um sistema parcialmente ergativo e parcialmente nominativo. In: A. D. Rodrigues, A. S. A. C. Cabral (orgs.), Linguas e culturas Macro-Jê. Brasília: Editora Universidade de Brasília, pp. 145-152.

Souza, Sueli Maria de. 1997. A sintaxe de uma língua de verbo no final: Krahô. Tese de doutorado, Universidade de São Paulo.

Urban, Greg. 1985. Ergativity and accusativity in Shokleng. International Journal of American Linguistics, 51.2:164-187.

Wiesemann, Ursula. 1986. The pronoun systems of some Jê and Macro-Jê languages. In: Ursula Wiesemann (ed.), Pronominal systems. Tübingen: Gunther Narr Verlag, pp. 359-380 